\section{A machine learning model for the differentiation of hydrous magma at lower crust}

BODA LIU, CIN-TY LEE AND RAJDEEP DASGUPTA

Rice University

Presenting Author: boda_liu@hotmail.com

The fundamental question of how magma evolves during its ascent through the crust remains poorly understood. To the firstorder, the continental crust consists of mafic lower crust and felsic to intermediate upper crust. The lower crust cumulates are consistent with mineral assemblages produced by crystal fractionation experiments of hydrous magma at lower crust pressures (Müntener and Ulmer, 2018). However, the bulk composition of the upper crust is constantly missed by the resulted melt in crystal fractionation experiments. We hypothesize that the incomplete melt-cumulates separation in the lower crust could explain the compositional trend observed in upper crust.

We consider mixing the derived melt with cumulates to generate a new parental magma for upper crust series. This new magma then follows specific cooling path and evolves to more felsic compositions. We use a machine learning model to keep track of the composition of the melt and the mineral assemblage. The data to train the machine learning model include LEPR and some recent studies. We use multiple geochemical proxies to check the equilibrium of experimental runs. We also add or remove phases in equilibrium assemblage to synthesize new bulk compositions to feed the data-hungry machine learning algorithm. We use a simple three-layer neural network architecture and train the model on a NVIDIA 3060Ti GPU. The machine learning model is designed to reproduce the melt composition and mineral assemblage given bulk composition, pressure, temperature, oxygen fugacity, and water content. Compared to the thermodynamic model MELTS, the machine learning model is much better in fitting melt compositions and proportions of minerals.

I will continue to develop and maintain the software, "Marmalade" (Magma Remake with Machine Learning and Data from Experiments) in accessible programming language (e.g., Excel spreadsheet and Python). The software will have customizable features pertaining to realistic crustal magmatic processes including polybaric fractionation and incomplete crystal-melt separation.

Reference: Müntener, O., Ulmer, P., 2018. Arc crust formation and differentiation constrained by experimental petrology. American Journal of Science 318, 64-89.
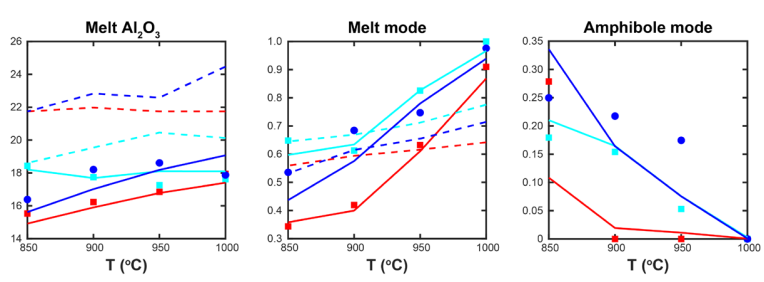

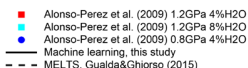

